И.А. Рудаков, А.П. Лукавый

\title{
ПЕРИОДИЧЕСКИЕ РЕШЕНИЯ КВАЗИЛИНЕЙНОГО ВОЛНОВОГО УРАВНЕНИЯ С ПЕРЕМЕННЫМИ КОЭФФИЦИЕНТАМИ
}

Доказаны теоремы существования периодических по времени решений квазилинейного волнового уравнения с непостоянными коэффициентами и однородными граничными условиями, одно из которых является условием Неймана.

Ключевые слова: волновое уравнение, периодические решения, задача Штурма-Лиувилля, ряд Фурье.

Исследуется задача о периодических решениях волнового уравнения

$$
\begin{aligned}
& p(x) u_{t t}-\left(p(x) u_{x}\right)_{x}=g(x, t, u)+f(x, t), 0<x<\pi, t \in R ; \\
& u(x, t+T)=u(x, t), \quad 0<x<\pi, \quad t \in R ; \\
& u_{x}(0, t)=u(\pi, t)+h u_{x}(\pi, t)=0, \quad t \in R .
\end{aligned}
$$

Уравнение (1) является нелинейной моделью распространения волн в неизотропной среде [1]. Уравнение более общего вида

$$
\rho(z) u_{t t}-\left(\mu(z) u_{z}\right)_{z}=h(z, t, u)+F(z, t),
$$

описывающее распространение сейсмических волн, приводится к уравнению (1) с помощью замены переменной $x=\int_{0}^{z} \sqrt{\frac{\rho(s)}{\mu(s)}} d s$. Здесь $\mu(z)$ - коэффициент эластичности, $\rho(z)$ плотность породы, $p=\sqrt{\rho \mu}$ - акустический импеданс [1].

Задача о периодических решениях волнового уравнения с постоянными коэффициентами $(p(x) \equiv 1)$ исследовалась в работах [2-7]. В работах $[1 ; 8-10]$ изучалось квазилинейное волновое уравнение с переменными коэффициентами. В [1; 8] доказано существование по крайней мере одного [1] или счетного числа [8] периодических решений в случае однородных граничных условий Дирихле. В работе [9] доказано существование по крайней мере одного периодического решения при произвольных однородных граничных условиях, если нелинейное слагаемое имеет степенной рост. В [10] доказано существование бесконечного числа периодических по времени решений в случае граничных условий третьего рода и Дирихле. Целью данной работы является доказательство теорем о существовании и регуляризации либо бесконечного числа периодических решений для волнового уравнения с переменными коэффициентами и однородными граничными условиями, одно из которых является условием Неймана, если нелинейное слагаемое имеет степенной рост, либо хотя бы одного решения, когда нелинейное слагаемое удовлетворяет условию нерезонансности на бесконечности.

Если в (3) граничные условия при $x=0$ и $x=\pi$ поменять местами, то получим эквивалентную задачу, поскольку замена $x=\pi-y, u(x, t)=u(\pi-y, t)$ переставляет местами концы отрезка $[0, \pi]$.

Пусть функция $p(x)$ удовлетворяет следующим условиям [1]:

$$
p(x) \in C^{2}[0, \pi], p(x) \geq d>0 \quad \forall x \in[0, \pi], \quad \rho=\min _{[0, \pi]} \eta_{p}(x)>0,
$$

где $\eta_{p}(x)=\frac{1}{2} \frac{p^{\prime \prime}}{p}-\frac{1}{4}\left(\frac{p^{\prime}}{p}\right)^{2}$. В качестве примера такой функции можно взять 
где $\alpha, C_{1}, C_{2}>0$.

$$
p(x)=\left(C_{1} x+C_{2}\right)^{2+\alpha}
$$

В работе будут рассмотрены два случая. В случае I, когда

$$
h=0 \text {, }
$$

потребуем выполнения неравенства

$$
p^{\prime}(0) \geq 0 \text {. }
$$

В случае II, когда в граничных условиях (3) имеем $h>0$, потребуем выполнения (7), а также неравенства

$$
\frac{p^{\prime}(\pi)}{p(\pi)} \leq \frac{2}{h}
$$

Если $p(x)$ имеет вид (5), то условие (8) выполнено, когда $\frac{C_{2}}{C_{1}} \geq\left(1+\frac{\alpha}{2}\right) h-\pi$.

Свойства волнового оператора. Решения задач (1)-(3); (1),(2),(4) будем искать в виде ряда Фурье. Для построения ортонормированной системы рассмотрим задачу ШтурмаЛиувилля:

$$
\begin{gathered}
-\left(p(x) \varphi^{\prime}(x)\right)^{\prime}=\lambda p(x) \varphi(x) ; \\
\varphi^{\prime}(0)=\varphi(\pi)+h \varphi^{\prime}(\pi)=0 .
\end{gathered}
$$

Стандартно доказывается [11], что при $h \geq 0$ задача (9), (10) имеет простые положительные собственные значения $\lambda=\lambda_{n}^{2}, n \in N$. Обозначим $\varphi_{n}(x)$ собственные функции задачи (9), (10), соответствующие собственным значениям $\lambda_{n}^{2}$.

Рассмотрим пространство $L_{2}(0, \pi)$ со скалярным произведением

$$
(\varphi, \psi)=\int_{[0, \pi]} p(x) \varphi(x) \psi(x) d x, \quad \varphi, \psi \in L_{2}(0, \pi) .
$$

После нормировки в $L_{2}(0, \pi)$ система функций $\left\{\varphi_{n}(x)\right\}_{n=1}^{\infty}$ станет ортонормированной и полной в $L_{2}(0, \pi)[12]$.

Для изучения асимптотики $\lambda_{n}$ сделаем стандартную замену переменной $z(x)=\sqrt{p(x)} \varphi(x)$. Тогда соотношению (9) будет соответствовать равенство

$$
z^{\prime \prime}+\left(\lambda-\eta_{p}(x)\right) z=0 .
$$

В случаях I, II для функции $z$ будут выполнены соответственно следующие граничные условия:

$$
\begin{aligned}
& z^{\prime}(0)-z(0) h_{1}=z(\pi)=0 \\
& z^{\prime}(0)-z(0) h_{1}=z^{\prime}(\pi)+h_{2} z(\pi)=0 .
\end{aligned}
$$

Здесь $\quad h_{1}=\frac{p^{\prime}(0)}{2 p(0)}, h_{2}=\frac{2 p(\pi)-h p^{\prime}(\pi)}{2 h p(\pi)}$.

Случай I разобьем на два подслучая. Если $p^{\prime}(0)>0$, то $h_{1}>0$ и доказано [10] представление

$$
\lambda_{n}=n-\frac{1}{2}+\theta_{n}
$$

в котором для последовательности $\theta_{n}$ справедливы оценки

$$
0<b_{0} \frac{1}{n} \leq \theta_{n} \leq b_{1} \frac{1}{n} \quad \forall n \in N
$$

Константы $b_{0}, b_{1}$ не зависят от $n$. Если $p^{\prime}(0)=0$, то $h_{1}=0$ и для $\lambda_{n}$ также справедливы представление (13) и оценка (14) [9]. 
Рассмотрим случай II. Если $p^{\prime}(0)=0$ и $\frac{p^{\prime}(\pi)}{p(\pi)}=\frac{2}{h}$, то $z(x)$ удовлетворяет условиям

Тогда $\lambda_{n}$ выражается формулой [9]

$$
z^{\prime}(0)=z^{\prime}(\pi)=0 .
$$

$$
\lambda_{n}=n-1+\theta_{n} .
$$

Для последовательности $\theta_{n}$ также справедлива оценка (14), в которой константы $b_{0}, b_{1}$ не зависят от $n$.

Пусть в случае II имеют место равенство $p^{\prime}(0)=0$ и неравенство $\frac{p^{\prime}(\pi)}{p(\pi)}<\frac{2}{h}$. Тогда из (12) получим соотношение

$$
z^{\prime}(0)=z^{\prime}(\pi)+h_{2} z(\pi)=0 .
$$
ми

Следуя плану из работы [10], рассмотрим уравнение с постоянными коэффициента-

$$
z^{\prime \prime}+\bar{\lambda} z=0
$$

При $\bar{\lambda}=0$ задача (16), (17) имеет единственное решение $z \equiv 0$. При $\bar{\lambda} \neq 0$ имеет место равенство $\bar{\lambda} \int_{0}^{\pi} z^{2}(x) d x=\int_{0}^{\pi}\left(z^{\prime}(x)\right)^{2} d x+h_{2} z^{2}(\pi)$. Поэтому $\bar{\lambda}=k^{2}, k>0$, и задача $(16),(17)$ имеет решение $z=\cos (k x)$. Отсюда и из равенства (16) получим уравнение

$$
\operatorname{ctg}(k \pi)=h_{0} k \text {, }
$$

в котором $h_{0}=1 / h_{2}$. Элементарные рассуждения показывают, что положительные решения уравнения (18) имеют вид

$$
k=n-1+\bar{\theta}_{n}, n \in N
$$

и $\theta_{n} \in(0,1 / 2)$. Подставив (19) в (18), получим уравнение

$$
\bar{\theta}_{n}=\frac{1}{\pi h_{0}} \frac{1}{n} f_{1}\left(\pi \bar{\theta}_{n}\right) \frac{1}{1-\left(1-\bar{\theta}_{n}\right) / n} .
$$

Здесь $f_{1}(t)=\frac{t}{\operatorname{tg} t}$. Функция $f_{1}$ убывает на промежутке $\left(0, \frac{\pi}{2}\right)$, и $\lim _{t \rightarrow 0} f_{1}(t)=1$. Отсюда и из (20) вытекает существование констант $c_{0}, c_{1}$, таких, что

$$
0<c_{0} \frac{1}{n} \leq \bar{\theta}_{n} \leq c_{1} \frac{1}{n} \quad \forall n \in N .
$$

Обозначим $\eta=\max _{t \in[0, \pi]} \eta_{p}(x)$ и рассмотрим уравнения

$$
\begin{aligned}
& z^{\prime \prime}+(\lambda-\eta) z=0 ; \\
& z^{\prime \prime}+(\lambda-\rho) z=0 .
\end{aligned}
$$

Пусть $\left\{\lambda_{n}^{\prime}\right\},\left\{\lambda_{n}^{\prime \prime}\right\},\left\{\bar{\lambda}_{n}\right\}$ суть последовательности собственных значений задач Штурма-Лиувилля (23), (16); (22),(16); (17),(16). Из теорем сравнения [11] следуют неравенства

$$
\lambda_{n}^{\prime} \leq \lambda_{n}^{2} \leq \lambda_{n}^{\prime \prime}
$$

По доказанному $\lambda_{n}^{\prime}-\rho=\left(n-1+\bar{\theta}_{n}\right)^{2}, \lambda_{n}^{\prime \prime}-\eta=\left(n-1+\bar{\theta}_{n}\right)^{2}$. Отсюда и из (21), (24) вытекает существование последовательности, которую также обозначим $\theta_{n}$, и не зависящих от $n$ констант $b_{0}, b_{1}$, для которых выполнены соотношение (15) и неравенства (14). 
Пусть в случае II имеют место неравенство $p^{\prime}(0)>0$ и равенство $\frac{p^{\prime}(\pi)}{p(\pi)}=\frac{2}{h}$. Тогда из (12) получим соотношение $z^{\prime}(0)-h_{1} z(0)=z^{\prime}(\pi)=0$. В этом случае для $\lambda_{n}$ также имеют место представление (15) и оценки (14), поскольку замена переменной $x=\pi-y, u(x, t)=u(\pi-y, t)$ приведет к предыдущему случаю.

Если в случае II имеют место строгие неравенства $p^{\prime}(0)>0, \frac{p^{\prime}(\pi)}{p(\pi)}<\frac{2}{h}$, то в граничных условиях (12) будем иметь $h_{1}>0, h_{2}>0$. В [10] доказано, что и в этом случае справедливы равенство (15) и неравенства (14).

Таким образом, в случае I $\lambda_{n}$ имеют представление (13), а в случае II - представление (15), где для $\theta_{n}$ справедливы неравенства (14). Заметим, что поскольку

$$
\int_{0}^{\pi} p(x) \varphi_{n}^{\prime}(x) \varphi_{m}^{\prime}(x) d x=p(\pi) \varphi_{n}^{\prime}(\pi) \varphi_{m}(\pi)-p(0) \varphi_{n}^{\prime}(0) \varphi_{m}(0),
$$

то в случае I последовательность $\left\{\varphi_{n}^{\prime}(x)\right\}$ является ортогональной в $L_{2}(0, \pi)$.

Будем искать периодические решения с периодом времени, имеющим следующий вид:

$$
T=2 \pi \frac{b}{a}, \quad a, b \in N, \text { НОД }(a, b)=1 .
$$

Обозначим $\Omega=[0, \pi] \times R \backslash(T Z)$ и рассмотрим пространство $L_{2}(\Omega)$, скалярное произведение и норма в котором задаются формулами

$$
(f, g)=\int_{\Omega} f(x, t) g(x, t) p(x) d x d t, \quad\|f\|^{2}=\int_{\Omega} f^{2}(x, t) p(x) d x d t \quad \forall f, g \in L_{2}(\Omega) .
$$

Рассмотрим полную, ортонормированную в $L_{2}(\Omega)$ систему функций

$$
\Lambda=\left\{\frac{1}{\sqrt{T}} \varphi_{n}(x), \sqrt{\frac{2}{T}} \varphi_{n}(x) \cos \left(\frac{a}{b} m t\right), \sqrt{\frac{2}{T}} \varphi_{n}(x) \sin \left(\frac{a}{b} m t\right)\right\}_{m, n \in N} .
$$

Определим оператор $A_{0}: L_{2}(\Omega) \rightarrow L_{2}(\Omega)$, для которого

$$
D\left(A_{0}\right)=\left\{u \in C^{2}(\Omega)\left|u=\sum_{n=1}^{N} \sum_{m=0}^{M} \varphi_{n}(x)\left(a_{n m} \cos \left(\frac{a}{b} m t\right)+b_{n m} \sin \left(\frac{a}{b} m t\right)\right)\right| N, M \in \mathrm{N}\right\}
$$

и $\quad A_{0} \varphi=p \varphi_{t t}-\left(p \varphi_{x}\right)_{x} \quad \forall \varphi \in D\left(A_{0}\right)$. Пусть $\quad \bar{A}_{0} \varphi=\frac{1}{p} A_{0} \varphi \quad \forall \varphi \in D\left(A_{0}\right)$. Множество функций $D\left(A_{0}\right)=D\left(\bar{A}_{0}\right)$ всюду плотно в $L_{2}(\Omega)$. Обозначим буквой $A$ оператор в $L_{2}(\Omega)$, являющийся замыканием по графику оператора $\bar{A}_{0}$. Система функций $\Lambda$ является системой собственных функций операторов $\bar{A}_{0}$ и $A$ с собственными значениями $\mu_{n m}=\lambda_{n}^{2}-\left(\frac{a}{b} m\right)^{2}, \quad n \in N, m \in N \bigcup\{0\}$. Для оператора $A$ справедливы следующие свойства [10]: 1) $A$ самосопряжен, $\left.\left.\quad A^{-1} \in L(R(A), R(A)) ; \quad 2\right) \quad \operatorname{dim} N(A)<+\infty ; 3\right)$ $L_{2}(\Omega)=N(A) \oplus R(A)$.

Пусть $\bar{H}_{k}(\Omega), H_{k}(\Omega), k \in\{1,2\}$, есть пространства Соболева, полученные замыканием соответственно $D\left(A_{0}\right), C^{\infty}(\Omega)$ по норме $\|u\|_{k}^{2}=\sum_{|\sigma|=0}^{k}\left\|D^{\alpha} u\right\|^{2}$, где 


$$
\sigma=\left(\sigma_{1}, \sigma_{2}\right) \in Z_{+} \times Z_{+}, Z_{+}=N \bigcup\{0\},|\sigma|=\sigma_{1}+\sigma_{2}, D^{\alpha}=\frac{\partial^{|\sigma|}}{\partial x^{\sigma_{1}} \partial t^{\sigma_{2}}} .
$$

Лемма 1. В случае I при нечетном $b$ для любой функции $u \in L_{2}(\Omega) \cap R(A)$ имеют место включение $A^{-1} u \in C(\Omega) \cap H^{1}(\Omega)$ и неравенство $\left\|A^{-1} u\right\|_{1} \leq C\|u\|$, где константа $C$ не зависит от $u$.

Доказательство. Произвольную функцию $u \in L_{2}(\Omega) \bigcap R(A)$ представим в виде суммы ряда Фурье по системе (26):

$$
u=\sum_{\mu_{n n} \neq 0} \varphi_{n}(x)\left(a_{n m} T_{m} \cos \left(\frac{a}{b} m t\right)+b_{n m} T_{m} \sin \left(\frac{a}{b} m t\right)\right) .
$$

Здесь $T_{m}= \begin{cases}1 / \sqrt{T}, & m=0 ; \\ \sqrt{2 / T}, & m \neq 0 .\end{cases}$

Пусть $v=A^{-1} u$. Тогда

$$
\nu=\sum_{\mu_{n m} \neq 0} \frac{1}{\mu_{n m}} \varphi_{n}(x)\left(a_{n m} T_{m} \cos \left(\frac{a}{b} m t\right)+b_{n m} T_{m} \sin \left(\frac{a}{b} m t\right)\right) .
$$

Докажем, что

$$
v_{t}=\frac{a}{b} \sum_{\mu_{n m} \neq 0} \frac{m}{\mu_{n m}} \varphi_{n}(x)\left(-a_{n m} T_{m} \sin \left(\frac{a}{b} m t\right)+b_{n m} T_{m} \cos \left(\frac{a}{b} m t\right)\right) .
$$

В случае I имеем

$$
\left|\mu_{n m}\right|=\left|n-\frac{1}{2}-\frac{a}{b} m+\theta_{n}\right| \cdot\left|n-\frac{1}{2}+\frac{a}{b} m+\theta_{n}\right| \geq \frac{1}{4 b}\left|(2 n-1) b-2 a m+2 b \theta_{n}\right| \cdot\left|n+\frac{a}{b} m\right| .
$$

Если $b$-нечетное число, то $|(2 n-1) b-2 a m| \geq 1$ и, учитывая (14), при $\mu_{n m} \neq 0$ выведем существование константы $\alpha_{0}>0$, такой, что

$$
\left|\mu_{n m}\right| \geq \alpha_{0}(n+m) \text {. }
$$

Обозначим $\quad h=\frac{a}{b} \sum_{\mu_{n m} \neq 0} \frac{m}{\mu_{n m}} \varphi_{n}(x)\left(-a_{n m} T_{m} \sin \left(\frac{a}{b} m t\right)+b_{n m} T_{m} \cos \left(\frac{a}{b} m t\right)\right)$,

$$
v_{N}=\sum_{n, m \leq N} \frac{1}{\mu_{n m}} \varphi_{n}(x)\left(a_{n m} T_{m} \cos \left(\frac{a}{b} m t\right)+b_{n m} T_{m} \sin \left(\frac{a}{b} m t\right)\right) .
$$

Из (28) следует включение $h \in L_{2}(\Omega)$. Легко видеть, что $v_{N} \rightarrow v,\left(v_{N}\right)_{t} \rightarrow h \quad$ в $L_{2}(\Omega) \quad$ при $\quad N \rightarrow \infty$. Отсюда следует (27) и $\left\|v_{t}\right\|^{2} \leq \frac{a^{2}}{\alpha_{0}^{2} b^{2}} \sum_{\mu_{n m} \neq 0}\left(a_{n m}^{2}+b_{n m}^{2}\right)=\frac{a^{2}}{\alpha_{0}^{2} b^{2}}\|u\|^{2}$. Интегрируя $\quad$ по $\quad$ частям, вычислим $\int_{0}^{\pi}\left(\varphi_{n}^{\prime}(x)\right)^{2} p(x) d x=\lambda_{n}^{2}$. Кроме того, в случае I при $n \neq m$ имеет место равенство 0 $\int_{0}^{\pi} \varphi_{n}^{\prime}(x) \varphi_{m}^{\prime}(x) p(x) d x=0$. Поэтому система функций

$$
\left\{\frac{\varphi_{n}^{\prime}(x)}{\lambda_{n}} T_{m} \cos \left(\frac{a}{b} m t\right), \frac{\varphi_{n}^{\prime}(x)}{\lambda_{n}} T_{m} \sin \left(\frac{a}{b} m t\right)\right\}_{n=1, m=0}^{\infty}
$$

является ортонормированной в $L_{2}(\Omega)$. Обозначим 


$$
g=\sum_{\mu_{n m} \neq 0} \frac{\lambda_{n}}{\mu_{n m}} \cdot \frac{\varphi_{n}^{\prime}(x)}{\lambda_{n}}\left(a_{n m} T_{m} \cos \left(\frac{a}{b} m t\right)+b_{n m} T_{m} \sin \left(\frac{a}{b} m t\right)\right) .
$$

Из (13), (14), (28) следует включение $g \in L_{2}(\Omega)$. Поскольку $\left(v_{N}\right)_{x} \rightarrow g, v_{N} \rightarrow v$ в $L_{2}(\Omega)$ при $N \rightarrow \infty$, то $v_{x}=g$. Кроме того, из (13), (14), (28) выведем оценку

$$
\left\|v_{x}\right\|^{2} \leq \frac{1}{\alpha_{0}^{2}} \sum_{\mu_{n m} \neq 0}\left(a_{n m}^{2}+b_{n m}^{2}\right)=\frac{1}{\alpha_{0}^{2}}\|u\|^{2} .
$$

Поскольку $\left|\varphi_{n}(x)\right| \leq C_{0} \forall x \in[0, \pi], \forall n \in N[11]$, то

$$
\begin{aligned}
& \sum_{\mu_{n m} \neq 0} \frac{1}{\left|\mu_{n m}\right|}\left|\varphi_{n}(x)\right|\left(\left|a_{n m}\right| T_{m}\left|\cos \left(\frac{a}{b} m t\right)\right|+\left|b_{n m}\right| T_{m}\left|\sin \left(\frac{a}{b} m t\right)\right|\right) \leq \\
\leq & C_{0} \sum_{\mu_{n m} \neq 0} \frac{1}{\left|\mu_{n m}\right|}\left(\left|a_{n m}\right|+\left|b_{n m}\right|\right) \leq C_{0} \sqrt{\sum_{\mu_{n m} \neq 0} \frac{1}{\left|\mu_{n m}\right|^{2}}} \sqrt{\left.\sum_{\mu_{n m} \neq 0}^{\sum\left(a_{n m}^{2}+b_{n m}^{2}\right.}\right)}<\infty .
\end{aligned}
$$

Сходимость ряда $\sum_{\mu_{n m} \neq 0} \frac{1}{\mu_{n n m}^{2}} \quad$ доказывается стандартно. Следовательно, $A^{-1} u \in C(\Omega) \cap H^{1}(\Omega)$. Лемма доказана.

Лемма 2. Для любой функции $u \in \bar{H}_{1}(\Omega) \bigcap R(A)$ имеют место включения $A^{-1} u \in C^{1}(\Omega),\left(A^{-1} u\right)_{t t},\left(A^{-1} u\right)_{t x} \in L_{2}(\Omega)$.

Доказательство. Возьмем произвольную функцию $u \in \bar{H}_{1}(\Omega) \cap R(A)$. Пусть $\left\{\psi_{l}\right\} \subset D\left(A_{0}\right)$ есть такая последовательность, что $\psi_{l} \rightarrow u,\left(\psi_{l}\right)_{x} \rightarrow u_{x},\left(\psi_{l}\right)_{t} \rightarrow u_{t}$ в $L_{2}(\Omega)$ при $l \rightarrow \infty$. Интегрируя по частям, получим

$$
\int_{\Omega} \psi_{l} \varphi_{n}(x) \cos \left(\frac{a}{b} m t\right) p(x) d x d t=-\frac{b}{a m} \int_{\Omega}\left(\psi_{l}\right)_{t} \varphi_{n}(x) \sin \left(\frac{a}{b} m t\right) p(x) d x d t .
$$

Переходя в этом равенстве к пределу при $l \rightarrow+\infty$, получим

$$
\int_{\Omega} u \varphi_{n}(x) \cos \frac{a}{b} m t p(x) d x d t=-\frac{b}{a m} \int_{\Omega} u_{t} \varphi_{n}(x) \sin \frac{a}{b} m t p(x) d x d t .
$$

Пусть $a_{n m}^{\prime}, b_{n m}^{\prime}$ есть коэффициенты Фурье функции $u_{t}$ по системе (26). Из (30) следует, что $a_{n m}=-\frac{b}{a m} b_{n m}^{\prime}$. Аналогично доказывается, что $b_{n m}=\frac{b}{a m} a_{n m}^{\prime}$. Поскольку $u_{t} \in L_{2}(\Omega)$, то $\sum_{\mu_{n m} \neq 0}\left(\left(a_{n m}^{\prime}\right)^{2}+\left(b_{n m}^{\prime}\right)^{2}\right)<\infty$.

Следовательно, $\sum_{\mu_{n m} \neq 0} m^{2}\left(a_{n m}^{2}+b_{n m}^{2}\right)=\frac{b^{2}}{a^{2}} \sum_{\mu_{n m} \neq 0}\left(\left(a_{n m}^{\prime}\right)^{2}+\left(b_{n m}^{\prime}\right)^{2}\right)<\infty$.

Обозначим $v=A^{-1} u$. Из оценки

$$
\left\|v_{t t}\right\|^{2}=\frac{a^{4}}{b^{4}} \sum_{M} \frac{m^{4}}{\mu_{n m}^{2}}\left(a_{n m}^{2}+b_{n m}^{2}\right)=\frac{a^{4}}{b^{4}} \sum_{M} \frac{m^{2}}{\mu_{n m}^{2}} m^{2}\left(a_{n m}^{2}+b_{n m}^{2}\right) \leq \frac{a^{6}}{b^{6} \alpha_{0}^{2}}\|u\|_{1}^{2}<\infty
$$

следует включение $u_{t t} \in L_{2}(\Omega)$.

Докажем, что $v_{x t} \in L_{2}(\Omega)$. Для этого рассмотрим уравнение $A \omega=u_{t}$ и докажем, что $\omega=v_{t}$. По доказанному 


$$
u_{t}=\frac{a}{b} \sum_{\mu_{n m} \neq 0} T_{m} m \varphi_{n}(x)\left(-a_{n m} \sin \frac{a}{b} m t+b_{n m} \cos \frac{a}{b} m t\right) .
$$

Поэтому $\omega=\frac{a}{b} \sum_{\mu_{n m} \neq 0} \frac{T_{m} m}{\mu_{n m}} \varphi_{n}(x)\left(-a_{n m} \sin \frac{a}{b} m t+b_{n m} \cos \frac{a}{b} m t\right)$. Таким образом, $\omega=A^{-1} u_{t}=v_{t}$, а так как $u_{t} \in L_{2}(\Omega)$, то, согласно лемме 1 , имеем $v_{t x}=\omega_{x} \in L_{2}(\Omega)$ и $\|v\|_{t x} \leq C\left\|u_{t}\right\| \leq A_{0}\|u\|_{1}$. Константа $A_{0}$ не зависит от $u$.

Интегрируя по частям по $x$, получим

$$
\begin{aligned}
& \int_{\Omega} \psi_{l} \varphi_{n}(x) \cos \left(\frac{a}{b} m t\right) p(x) d x d t=-\frac{1}{\lambda^{2}} \int_{0}^{T} \cos \left(\frac{a}{b} m t\right) d t \int_{0}^{\pi} \psi_{l}\left(-\lambda_{n}^{2} \varphi_{n}(x) p(x)\right) d x= \\
= & -\frac{1}{\lambda_{n}^{2}} \int_{0}^{T} \cos \left(\frac{a}{b} m t\right) d t \int_{0}^{\pi} \psi_{l}\left(\varphi_{n}^{\prime}(x) p(x)\right)^{\prime} d x=\frac{1}{\lambda_{n}^{2}} \int_{\Omega}\left(\psi_{l}\right)_{x}^{\prime} \varphi_{n}^{\prime}(x) \cos \left(\frac{a}{b} m t\right) p(x) d x d t .
\end{aligned}
$$

Переходя в этом равенстве к пределу при $l \rightarrow+\infty$, получим

$$
\int_{\Omega} u \varphi_{n}(x) \cos \left(\frac{a}{b} m t\right) p(x) d x d t=\frac{1}{\lambda_{n}} \int_{\Omega} u_{x} \frac{\varphi_{n}^{\prime}(x)}{\lambda_{n}} \cos \left(\frac{a}{b} m t\right) p(x) d x d t .
$$

Обозначим $c_{n m}, d_{n m}$ коэффициенты Фурье функции $u_{x}$ по ортонормированной системе (29). Из последнего соотношения следует равенство $c_{n m}=\lambda_{n} a_{n m}$. Аналогично доказывается, что $d_{n m}=\lambda_{n} b_{n m}$. Поскольку $u_{x} \in L_{2}(\Omega)$, то $\underset{\mu_{n m} \neq 0}{\sum}\left(\left(c_{n m}\right)^{2}+\left(d_{n m}\right)^{2}\right)<\infty$. Следовательно, $\sum_{\mu_{n m} \neq 0} \lambda_{n}^{2}\left(\left(a_{n m}\right)^{2}+\left(b_{n m}\right)^{2}\right)<\infty$ и $\sum_{\mu_{n m} \neq 0} n^{2}\left(\left(a_{n m}\right)^{2}+\left(b_{n m}\right)^{2}\right)<\infty$. В [11] доказана оценка $\left|\varphi_{n}^{\prime}(x)\right| \leq B_{0} n \forall x \in[0, \pi]$. Следовательно,

$$
\begin{aligned}
& \sum_{\mu_{n m} \neq 0} \frac{1}{\left|\mu_{n m}\right|}\left|\varphi_{n}^{\prime}(x)\right|\left(\left|a_{n m}\right| T_{m}\left|\cos \left(\frac{a}{b} m t\right)\right|+\left|b_{n m}\right| T_{m}\left|\sin \left(\frac{a}{b} m t\right)\right|\right) \leq \\
\leq & B_{0} \sum_{\mu_{n m} \neq 0} \frac{n}{\left|\mu_{n m}\right|}\left(\left|a_{n m}\right|+\left|b_{n m}\right|\right) \leq B_{0} \sqrt{\sum_{\mu_{n m} \neq 0} \frac{1}{\mu_{n m}^{2}}} \cdot \sqrt{\sum_{\mu_{n m} \neq 0} n^{2}\left(a_{n m}^{2}+b_{n m}^{2}\right)}<\infty .
\end{aligned}
$$

Из сходимости ряда $\sum_{\mu_{n m} \neq 0}^{2}\left(a_{n m}^{2}+b_{n m}^{2}\right)$ аналогично доказывается неравенство $\sum_{\mu_{n m} \neq 0} \frac{m}{\left|\mu_{n m}\right|}\left(\left|a_{n m}\right|+\left|b_{n m}\right|\right)<\infty$. Из полученных оценок вытекает включение $v \in C^{1}(\Omega)$. Лемма доказана.

Квазилинейное уравнение. Обозначим $\sigma(A)=\left\{\mu_{n m} \mid n \in N, m \in Z_{+}\right\}$. Предположим, что функция $g$ удовлетворяет следующему условию:

$$
\alpha \leq \frac{g(x, t, u)}{u} \leq \beta, \text { если }|u| \geq C,(x, t) \in \Omega .
$$

Здесь константы $\alpha, \beta$ такие, что $\alpha \leq \beta$ и $C>0$.

Определение. Обобщенным решением задачи (1)-(3) называется функция $u \in L_{2}(\Omega)$, такая, что $\int_{\Omega} u\left(p \varphi_{t t}-\left(p \varphi_{x}\right)_{x}\right) d x d t=\int_{\Omega}(g(x, t, u)+f) \varphi d x d t \quad \forall \varphi \in D\left(A_{0}\right)$.

Замечание. Если $g \equiv 0, h=0$, то, согласно лемме 2, для любой функции $f \in \bar{H}_{1}(\Omega) \cap R(A)$ задача (1)-(3) имеет единственное обобщенное решение $u \in C^{1}(\Omega)$. 
Tеорема 1. Пусть выполнены условия (4), (6), (7), (25) и $b$ является нечетным числом. Предположим, что функция $g$ является $T$-периодической по $t, g \in C(\Omega \times R)$ и удовлетворяет условию (31) с константами $\alpha, \beta, C$, такими, что $C>0, \alpha \leq \beta$ и $[\alpha, \beta] \cap \sigma(A)=\varnothing$. Тогда для любой функции $f \in L_{2}(\Omega)$ задача (1)-(3) имеет обобщенное решение $u \in H_{1}(\Omega) \cap C(\Omega)$.

Доказательство теоремы 1 вытекает из теоремы 1.1 в работе [13] и леммы 1.

Рассмотрим случай II и случай I для четных значений $b$. В этих случаях множество $\sigma(A)$ имеет предельную точку из отрезка $\left[b_{0}, 2 b_{1}+b_{1}^{2}\right]$ и для оператора $A$ выполнено свойство II из работы [10]. Запишем уравнение (1) в виде

$$
p(x) u_{t t}-\left(p(x) u_{x}\right)_{x}+g(x, t, u)=f(x, t), 0<x<\pi, \quad t \in R .
$$

Следствием теоремы 3.2 из работы [10] является следующая теорема.

Tеорема 2. Пусть либо $h>0$ и выполнены условия (7), (8), либо $h=0$, выполнено условие (7) и $b$ является четным числом. Предположим, что функция $g$ является $T$ периодической по $t, g \in C(\Omega \times R)$ и удовлетворяет условию (31) с константами $\alpha, \beta, C$, такими, что $C>0,0<\alpha \leq \beta$ и $[-\beta,-\alpha] \cap \sigma(A)=\varnothing$. Пусть дополнительно производная $g_{u}(x, t, u)$ непрерывна в $\Omega \times R$ и $g_{u}(x, t, u) \geq-b_{0} p(x) \quad \forall(x, t, u) \in \Omega \times R$. Тогда для любой правой части $f \in L_{2}(\Omega)$ задача (32), (2), (3) имеет обобщенное решение $u \in L_{2}(\Omega)$.

Рассмотрим случай, когда функция $g$ имеет более чем линейный рост по $u$. Запишем уравнение (1) в следующем виде:

$$
p(x) u_{t t}-\left(p(x) u_{x}\right)_{x}+g(x, t, u)=0,0<x<\pi, \quad t \in R .
$$

Предположим, что функция

$$
\begin{aligned}
& g \in C(\Omega \times R), T \text {-периодична по } t \text {, не убывает по } u \\
& \text { и } \quad A_{3}|u|^{r-1}-A_{4} \leq|g(x, t, u)| \leq A_{1}|u|^{r-1}+A_{2} \quad \forall(x, t) \in \Omega, u \in R,
\end{aligned}
$$

где $A_{1}, A_{2}, A_{3}, A_{4}, r$ есть положительные числа, такие, что

$$
r>2, \quad \frac{A_{3}}{2}>\frac{A_{1}}{r} .
$$

При $q>1$ определим норму в пространстве $L_{q}(\Omega)$ формулой $\|u\|_{q}=\left(\int_{\Omega}|u(x, t)|^{q} p(x) d x d t\right)^{1 / q}$.

Определение. Обобщенным решением задачи (33), (2), (3) называется $T$-периодическая по $t$ функция $u \in L_{r}(\Omega)$, такая, что

$$
\int_{\Omega} u A_{0} \varphi d x d t+\int_{\Omega} g(x, t, u) \varphi d x d t=0 \quad \forall \varphi \in D\left(A_{0}\right) .
$$

Точно так же, как теорема 3.1 в [14], доказывается следующая теорема.

Tеорема 3. Предположим, что выполнены условия (4), (25), (34), (35), (36) и либо $g(x, t,-u)=-g(x, t, u) \quad \forall(x, t) \in \Omega, u \in R$, либо функция $g$ не зависит от $t$. Пусть либо $h>0$ и выполнены условия (7), (8), либо $h=0$ и выполнено условие (7). Тогда $\forall d>0$ существует обобщенное решение $u \in L_{r}(\Omega)$ задачи (33), (2), (3), такое, что $\|u\|_{r} \geq d$. В случае I при нечетном $b$ обобщенное решение $u \in H_{1}(\Omega) \cap C(\Omega)$.

В доказанных теоремах приведены условия существования периодических по времени решений квазилинейного волнового уравнения с однородными граничными условиями на отрезке, одно из которых является условием Неймана. 


\section{СПИСОК ЛИТЕРАТУРЫ}

1. Barby, V. Periodic solutions to nonlinear one dimensional wave equation with $x$ - dependent coefficients/ V. Barby, N. H. Pavel // Trans. Amer. Math. Soc.-1997.-V. 349. - № 5.- P. 2035-2048.

2. Rabinowitz, P. Free vibration for a semilinear wave equation/ P. Rabinowitz//Comm. Pure Aple. Math.-1978.V. 31.- № 1.- P. 31-68.

3. Bahri, A. Periodic solutions of a nonlinear wave equation/A. Bahri, H. Brezis// Proc. Roy. Soc. Edinburgh Sect. A. - 1980.- V. 85. - P. 3130-320.

4. Brezis, H. Forced vibration for a nonlinear wave equations/ H. Brezis, L. Nirenberg //Comm. Pure Aple. Math.1978. - V. 31. - № 1.- P. 1-30.

5. Плотников, П. И. Существование счетного множества периодических решений задачи о вынужденных колебаниях для слабо нелинейного волнового уравнения/П. И. Плотников// Математический сборник. 1988.-T. 136(178).- № 4(8). - C. 546-560.

6. Feireisl, E. On the existence of periodic solutions of a semilinear wave equation with a superlinear forcing term/ E. Feireisl //Chechosl. Math. J.- 1988.-V. 38.- № 1.- Р.- 78-87.

7. Рудаков, И.А. Нелинейные колебания струны/ И.А. Рудаков//Вестн. МГУ. Сер. 1, матем., мех. - 1984.№ 2. - С. 9-13.

8. Рудаков, И. А. Периодические решения нелинейного волнового уравнения с непостоянными коэффициентами/ И. А. Рудаков //Математические заметки . -2004 . -Т . 76. - Вып. 3. - С. 427-438.

9. Shuguan, J. Time periodic solutions to a nonlinear wave equation with $x$ - dependet coefficients/ J. Shuguan//Calc. Var. -2008.-V. 32. - P. 137-153.

10. Рудаков, И.А. Периодические решения квазилинейного волнового уравнения с переменными коэффициентами/ И.А. Рудаков //Математический сборник. -2007.-Т. 198.- № 4(8). - С. 546-560.

11. Трикоми, Ф. Дифференциальные уравнения/ Ф.Трикоми. - М.: УРСС, 2003.-351 с.

12. Бабич, В.М. Ортогональные разложения и метод Фурье/ В.М. Бабич, Н.С. Григорьева. - Л.: Изд-во ЛГУ, 1983. - $239 \mathrm{c}$.

13. Рудаков, И.А. Нелинейные уравнения, удовлетворяющие условию нерезонансности/ И.А. Рудаков // Труды Семинара им. И.Г. Петровского. -2006. - Вып. 25. - С. 226-243.

14. Рудаков, И.А. Периодические решения нелинейного волнового уравнения с однородными граничными условиями/ И.А. Рудаков //Изв. РАН.- 2006.- № 1. - С. 1-10.

Материал поступил в редколлегию 23.06.14. 